\title{
Reaching Activity in the Medial Posterior Parietal Cortex of Monkeys Is Modulated by Visual Feedback
}

\author{
Annalisa Bosco, ${ }^{1}$ Rossella Breveglieri, ${ }^{1}$ Eris Chinellato, ${ }^{2}$ Claudio Galletti, ${ }^{1}$ and Patrizia Fattori ${ }^{1}$ \\ ${ }^{1}$ Dipartimento di Fisiologia Umana e Generale, Università di Bologna, I-40126 Bologna, Italy, and ${ }^{2}$ Robotic Intelligence Laboratory, Jaume I University, \\ Campus Riu Sec, 12071, Castellón de la Plana, Spain
}

Reaching and grasping an object is an action that can be performed in light, as well as in darkness. Area V6A is a visuomotor area of the medial posterior parietal cortex involved in the control of reaching movements. It contains reaching neurons as well as neurons modulated by passive somatosensory and visual stimulations. In the present work we analyze the effect of visual feedback on reaching activity of V6A neurons. Three macaques were trained to execute reaching movements in two conditions: in darkness, where only the reaching target was visible, and in full light, where the monkey also saw its own moving arm and the environment. Approximately $85 \%$ of $\mathrm{V} 6 \mathrm{~A}$ neurons (127/149) were significantly related to the task in at least one of the two conditions. The majority of task-related cells (69\%) showed reach-related activity in both visual conditions, some were modulated only in light (15\%), while others only in dark (16\%). The sight of the moving arm often changed dramatically the cell's response to arm movements. In some cases the reaching activity was enhanced and in others it was reduced or disappeared altogether. These neuronal properties may represent differences in the degree to which cells are influenced by feedback control versus feedforward movement planning. On average, reach-related modulations were stronger in light than in dark, a phenomenon similar to that observed in brain imaging experiments in the human medial posterior parietal cortex, a region likely homologous to macaque area V6A.

\section{Introduction}

Reaching and grasping an object is an action that we perform in light and in dark. In the dark, reaching movements rely on efferent copies of motor signals and proprioceptive reafferent signals from the moving limb. However, reaching in the light relies not only on efferent motor and reafferent proprioceptive signals but also on visual information about the target to be reached, the moving forelimb, and the surroundings (Carlton, 1981; Prablanc et al., 1986). In the absence of visual feedback, movement performance quickly deteriorates (Woodworth, 1899; Vince, 1948; Keele and Posner, 1968; Carlton, 1981; Meyer et al., 1988; MaWyatt and McKee, 2007).

The posterior parietal cortex (PPC) is thought to integrate efferent motor signals with reafferent proprioceptive and visual signals related to movement execution (Hyvärinen and Poranen, 1974; Mountcastle et al., 1975; Andersen et al., 1997). PPC may play a role in comparing anticipated and actual sensory feedback for the correct execution of goal-directed actions (Wolpert et al., 1998; Grafton, 2010; Shadmehr et al., 2010).

The target of our study is an area of the medial PPC located in the caudalmost part of superior parietal lobule (SPL), named V6A (Fig. $1 A)$. Many neurons in this cortical area are responsive to visual stim-

Received May 3, 2010; revised Aug. 12, 2010; accepted Sept. 13, 2010.

This research was supported by European Union Grant FP7-ICT-217077-EYESHOTS, and by Ministero dell'Università e della Ricerca and Fondazione del Monte di Bologna e Ravenna, Italy. We are grateful to Nicoletta Marzocchi and to Giacomo Placenti for help in the quantitative analyses.

Correspondence should be addressed to Patrizia Fattori, Dipartimento di Fisiologia Umana e Generale, Università di Bologna, Piazza di Porta San Donato 2, I-40126 Bologna, Italy. E-mail: patrizia.fattori@unibo.it.

DOI:10.1523/JNEUROSCI.2313-10.2010

Copyright $\odot 2010$ the authors $\quad 0270-6474 / 10 / 3014773-13 \$ 15.00 / 0$ uli (Galletti et al., 1996; 1999), to somatosensory stimulations of upper limbs (Breveglieri et al., 2002), and to active arm movements (Galletti et al., 1997; Fattori et al., 2001). The functional properties of V6A neurons clearly reflect (and are likely the functional consequence of) the anatomical connections of this area. In fact, V6A receives projections directly from dorsal premotor cortex (Matelli et al., 1998; Shipp et al., 1998; Marconi et al., 2001; Gamberini et al., 2009), and the extrastriate visual area V6 (Galletti et al., 2001). Both neuronal properties and anatomical connections of V6A indicate that visual, somatosensory and motor signals interact and possibly become integrated in V6A (Galletti et al., 2003).

In the past, we demonstrated that reaching movements performed in dark modulate the activity of V6A neurons (Fattori et al., 2001), and that V6A neurons are sensitive to arm position and to arm movement direction (Breveglieri et al., 2002; Fattori et al., 2005). In the present work we tested whether the reach-related activity and its spatial tuning are influenced by the presence of visual feedback. To answer this question, animals were trained in an instructed-delay task to reach visual targets at various locations in the light and in the dark. Reaching activity in the light condition may reflect a copy of efferent commands, as well as proprioceptive and visual afferent feedback, whereas in the dark condition reaching activity may reflect only somatosensory and/or movementrelated input. As expected, we encountered three types of neurons. Movement-related neurons displayed an increase in activity for reaching in the dark, and their activity was unchanged when reaching in the light. Visual-related neurons responded only to reaching in the light. Visuomovement neurons responded to reaching in the dark, and their responses was either enhanced or reduced during reaching in the light. The latter finding suggests a complex pattern of 
A



B

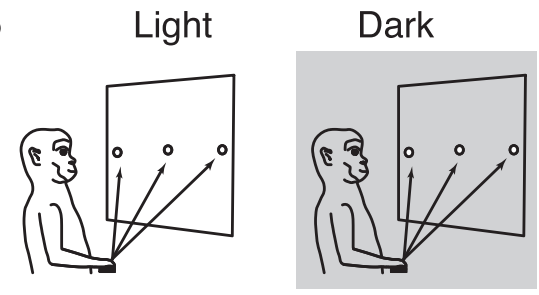

Figure 1. Recording site and reaching task. $\boldsymbol{A}$, Brain location of recording site. Part of the occipital pole and inferior parietal lobule has been cut away to show the location of the recording site (area V6A, gray shaded) in the anterior bank of the parieto-occipital sulcus. Locations of other parietal and frontal areas are also indicated. $\boldsymbol{B}$, Experimental setup. Reaching movements were performed in the light and in the dark (gray shaded) background, from a home button (black rectangle) toward one of three targets located on a panel in front of the animal. C, Time course of the task. The sequence of status of the home button (HB), target button (TB) and color of the target button (LED) are shown. Lower and upper limits of the time intervals of each epoch are indicated above the scheme. Below the scheme, typical examples of eye traces during a single trial is shown. $\boldsymbol{D}$, Time epochs during a typical trial. Short vertical ticks are the sequence of spikes collected from a neuron. Long vertical ticks among spikes indicate the occurrence of behavioral- or task-related events. From left to right, the markers indicate: trial start (HB press), target appearance (LED lit-on green), go signal for outward movement (LED red), start and end of outward reach movements (HB release and TB press, respectively), go signal for inward movement (LED switching off), start and end of the inward movement (TB release and HB press, respectively), end of data acquisition. The time epochs are indicated below the raster of spikes and markers.

firing modulation by the visual feedback, characterized by a nonadditive interaction between visual and somato-sensory/-motor-related signals.

Preliminary results have been previously presented in abstract form (Bosco et al., 2009).

\section{Materials and Methods}

Experiments were performed in accordance with Italian laws on care and use of laboratory animals and with the European Communities Council Directive of 24 November 1986 (86/609/EEC), and were approved by the Bioethical Committee of the University of Bologna. During training and recording sessions, particular care was taken to prevent behavioral and clinical signs of pain or distress.

Three trained male Macaca fascicularis were used in this work. They each sat in a primate chair and performed a reaching task. Single cell

activity was extracellularly recorded from the cortical area V6A (Fig. $1 \mathrm{~A}$ ) using glass-coated metal microelectrodes with a tip impedance of $0.8-2$ $\mathrm{M} \Omega$ at $1 \mathrm{kHz}$. Action potentials were sampled at $1 \mathrm{kHz}$ for two monkeys (Galletti et al., 1995) and at $100 \mathrm{kHz}$ for the third animal (Kutz et al., 2005). Eye movements were simultaneously recorded using an infrared oculometer (Dr. Bouis, Karlsruhe, Germany). They were sampled at 100 $\mathrm{Hz}$ for two monkeys (Galletti et al., 1995) and at $500 \mathrm{~Hz}$ for the third animal (Kutz et al., 2005). Eye position was monitored by an electronic window $\left(5 \times 5^{\circ}\right)$ centered on the fixation target.

Surgery to implant the recording apparatus was performed in asepsis and under general anesthesia (sodium thiopental, $8 \mathrm{mg} \cdot \mathrm{kg}^{-1} \cdot \mathrm{h}^{-1}$, i.v.). A full program of postoperative analgesia (ketorolac trometazyn, $1 \mathrm{mg} / \mathrm{kg}$, i.m. immediately after surgery, and $1.6 \mathrm{mg} / \mathrm{kg}$, i.m. on the following days) and antibiotic care (Ritardomicina, benzatinic benzylpenicillin + dihydrostreptomycin + streptomycin, 1-1.5 ml/10 kg every 5-6 d) followed surgery. Extracellular recording techniques and procedures to reconstruct microelectrode penetrations were similar to those described in other reports (Galletti et al., 1995; 1996). Area V6A was recognized on functional grounds following the criteria described by Galletti et al. (1999), and on anatomical grounds following the cytoarchitectonic criteria described by Luppino et al. (2005).

Reaching task. Monkeys performed arm movements with the contralateral limb, with the head restrained and while maintaining steady fixation. They performed reaching movements directed outward from the body (to reach a visual target) and backward toward the body (Fig. $1 B)$, similarly to natural reaches. The task was executed in two conditions: in the dark, such that the animal could only see the target, and in the light, such that the target, the moving arm, and the environment were visible. As shown in Figure $1 B$, reaching movements started from a button (home button, $2.5 \mathrm{~cm}$ in diameter) placed outside the animal's field of view, $5 \mathrm{~cm}$ in front of the chest on the midsagittal plane. Reaching movements transported the hand from the home button to targets positioned in different spatial locations on a frontoparallel panel located 14 $\mathrm{cm}$ from the eyes. Targets were light-emitting diodes (LEDs; $4 \mathrm{~mm}$ in diameter; $1.6^{\circ}$ of visual angle) mounted on microswitches embedded in the panel, with the central target placed straight ahead at eye level. We used two different arrangements of target positions. In one animal, targets were distributed in a $3 \times 3$ grid, $7 \mathrm{~cm}\left(28^{\circ}\right.$ visual angle $)$ apart along any row or column in the grid. In two animals, we used a panel with three targets distributed along a line, with spacing of 7.4 $\mathrm{cm}\left(30.8^{\circ}\right)$ apart from the other. This panel could be rotated around the central LED, to offer additional target locations.

The time sequence of the reaching task is shown in Figure 1C. A trial begins when the monkey presses the button near its chest. After button pressing the animal is free to look around and is not required to perform any eye or arm movements. After 200-1000 ms, one of the LEDs lights up (with green illumination). The monkey has to fixate the LED and wait for its change in color without performing any eye or arm movement. After a delay period of 500-2500 ms the LED color changes from green to red. This event represents the "go" signal for the monkey to release the button and to perform an arm-reaching movement to reach the LED and press it. The animal then keeps the hand on the LED until it switches off (after 500-1200 ms). This event cues the animal to release the LED and to return to the home button. The task ends with the home button press, which triggers the monkey's reward and the start of another trial.

The time sequence was the same in trials performed in dark and in light. In light, the animal saw the panel, and therefore the reaching targets, and its arm moving in the peripersonal space.

During the task, and for both task conditions, the monkey was required to fixate the target LED. If fixation was broken $\left(5^{\circ} \times 5^{\circ}\right.$ electronic window), trials were interrupted on-line and discarded. Trials where the monkey was fixating outside $\pm 0.5^{\circ}$ of the fixation point were discarded off-line after a trial-by-trial analysis.

The correct performance of reaching movements was monitored by pulses from microswitches (monopolar microswitches, RS Components) mounted under the home button and the LEDs. Button presses/ releases were recorded with $1 \mathrm{~ms}$ resolution. For a detailed description of the control system of trial execution, see Kutz et al. (2005). 
Data analysis. We compared the neural activity from light and dark conditions at different time epochs during the reaching task. Time epochs were defined as follows (Fig. $1 D$ ). FREE: from the beginning of the trial to the illumination of the LED (this epoch has been used as a reference period). DELAY: from beginning of fixation, after LED appearance, to the go signal cueing the onset of the reaching movement (LED change in color). MOV: from $200 \mathrm{~ms}$ before movement onset (home button release) to movement end (target button pressing). HOLD: from the end of forward reach (target button pressing) to $200 \mathrm{~ms}$ before backward movement onset (target button release). RET: from $200 \mathrm{~ms}$ before return movement onset (target button release) to movement end (home button pressing). MOV, HOLD and RET will be referred to as "action" epochs in this paper. A comparison of the neural activity during the action epochs with that during the "reference" epoch FREE was used to determine whether a neuron was significantly modulated by the arm-reaching movement. For those neurons that were tested with at least two target positions, we also evaluated the effect of the direction of arm movement. Given the high intrinsic variability in the activity of PPC cells, only cells with at least seven trials for each position were analyzed (Kutz et al., 2003).

We used a two-way ANOVA, where factor 1 was the epoch and factor 2 the arm direction. A post hoc test (Bonferroni-corrected $t$ test, $p<0.05$ ) was used to compare the activity during action epochs with that during epoch FREE. The effect of action epochs and arm direction on neural activity was considered significant in those cases where factor 1 and/or factor 2 and/or their interaction, and the post hoc test were significant $(p<0.05)$. FREE was chosen as a reference because in this epoch no visual stimuli were present, the monkey was free to look around, and it was not executing or preparing an arm movement. Only units displaying a significant task-related modulation in light, in dark, or in both conditions were further analyzed.

To quantify the spatial selectivity of recorded neurons in the two task conditions, we calculated a spatial index (SI) which takes into account the magnitude of the neuron response to each movement direction in each visual condition: $\mathrm{SI}=\left(n-\left(\sum r_{\mathrm{i}} / r_{\text {pref }}\right)\right) /(n-1)$, where $n$ is the number of spatial locations, $r_{\mathrm{i}}$ is the activity for the considered spatial location, and $r_{\text {pref }}$ is the activity for the preferred arm direction for the considered action epoch (MOV, HOLD, RET). The SI ranges between 0 and 1 . Neurons with value near 0 show the same magnitude of response for all reach directions, whereas neurons with value near 1 indicate a strict selectivity for one reach direction. SI was calculated for each neuron and for the two visual conditions. To compare the SI of the same cell for different visual conditions, confidence intervals on the preference indices were estimated using a bootstrap test. Synthetic response profiles were created by drawing $N$ firing rates (with replacement) from the $N$ repetitions of experimentally determined firing rates. The SI was recomputed using these $N$ firing rates. Ten thousand iterations were performed, and confidence intervals were estimated as the range that delimited $95 \%$ of the computed indices (Batista et al., 2007).

We quantified how the spatial sensitivity changes between epochs by computing the perpendicular distance (Dist) from the diagonal for each neuron: Dist $=\mid$ Spatial Index $($ Light $)-$ Spatial Index $(\operatorname{Dark}) \mid / \sqrt{2}$. Values range between 0 and 0.7 . Those values close to 0 (i.e., close to the diagonal) indicate that cells do not change their spatial sensitivity in the different epochs. Conversely, values close to 0.7 (i.e., far from the diagonal) indicate neurons that change their spatial sensitivity across epochs.

Significant modulation of neural activity by the direction of arm movement and visual conditions of the task was studied through a twoway ANOVA (factor 1: movement direction; factor 2: visual condition). The neural modulation relative to visual condition was assessed when factor 2 was significant $(p<0.05)$. The effect of visual condition on spatial tuning of reach-related activity was considered positive when factor 2 and interaction factor were significant $(p<0.05)$.

To assess the strength of visual modulation on reaching activity, we calculated an index [visual index (VI)]: VI = (best light - best dark)/ (best light + best dark), where best light and best dark are the mean average rates of discharge of each neuron in the light and in the dark, respectively, for the direction of arm movement evoking the best response in dark. The index ranges from -1 to 1 . A neuron whose reaching activity is elicited only in presence of visual feedback (i.e., in the light) will have a value of 1 , whereas a value of -1 denotes a neuron active only in dark. Values close to 0 indicate that the neuron is similarly modulated by reaching in light and dark conditions. We used the Cramer-Von Mises test to evaluate normality of the VI $(p<0.05)$.

Population responses of tested neurons were computed as average spike density functions (SDFs). A SDF was calculated (Gaussian kernel, half-width $40 \mathrm{~ms}$ ) for each neuron included in the analysis. SDF was averaged across all trials with the preferred reach directions, separately for light and dark conditions. The peak discharge rate of the neuron was used to normalize SDFs. The normalized SDFs were then averaged to derive population responses (Marzocchi et al., 2008). Population SDFs in dark and in light were compared with each other using a permutation test (10,000 iterations). Because the weighted mean of the forward movement time was $354 \pm 50 \mathrm{~ms}$, that of hold period was randomly varied between 800 and $1200 \mathrm{~ms}$, and that of backward movement was $233 \pm 67$ $\mathrm{ms}$, comparisons have been made in the following intervals: from $200 \mathrm{~ms}$ before movement onset to $300 \mathrm{~ms}$ after it for epoch MOV; from pressing of target button to $800 \mathrm{~ms}$ after it for epoch HOLD; from $200 \mathrm{~ms}$ before return onset to $200 \mathrm{~ms}$ after it for epoch RET. For the DELAY, comparisons in the permutation test have been made in the last $500 \mathrm{~ms}$ before the go signal, cueing the monkey to execute the reaching.

To further determine neural activity patterns that may have not been captured by the above statistical techniques, we performed a principal component analysis (PCA) on the data. PCA involves computing the covariance matrix of the data with respect to all dimensions of interest, and in extracting eigenvectors and eigenvalues of this matrix. The resulting eigenvectors constitute a different set of components that, linearly summed, allow reconstruction of the data. This analysis can provide further insight for data interpretation because each eigenvalue represents the weight of the corresponding component of the data, i.e., the amount of variance explained. Therefore, once the eigenvalues are normalized, they can be ranked based on their relative importance in capturing the variance of the whole dataset. If one or more components have eigenvalues very close to 0 , it means that some of the original dimensions can be computed from the others, and are thus redundant.

Here we performed a PCA of the responsiveness of all neurons and conditions, for each epoch of interest. Therefore, PCA was performed over the entire dataset of 116 neurons for each of the three epochs, to assess whether there was redundancy among the three original dimensions of the data (ipsilateral, central and contralateral position of the target with respect to the recording side), and to search for neural activity patterns that might not have been captured by the above statistical analysis.

For the neurons that show spatial tuning in the three horizontal target positions, we computed a preferred direction index (PD), in the two task conditions for each epoch of interest. This was done by calculating an average of the three possible positions weighted by their firing rates as follows: $\mathrm{PD}=\left(2 f r_{\text {contra }}+3 f r_{\text {center }}+4 f r_{\text {ipsi }}\right) /\left(f r_{\text {contra }}+f r_{\text {center }}+f r_{\text {ipsi }}\right)$, where $f r_{\text {contra }}$ is the average firing rate in the task epoch of interest in one visual condition for movements performed toward the contralateral target with respect to the recording side; $f r_{\text {center }}$ and $f r_{\text {ipsi }}$ are the average firing rates for movements directed to central and ipsilateral target positions, respectively. PD index ranges from 2 to 4 . Values near to 2, 3, or 4 indicate that the neuron shows preference for the contralateral, central, or ipsilateral target position, respectively.

All the analyses were performed using custom scripts in Matlab (MathWorks).

\section{Results}

We recorded the activity of 149 V6A neurons in three animals performing a reaching task toward different positions in peripersonal space, in light and dark conditions (Fig. $1 B, C$ ).

A quantitative evaluation of neural modulation (two-way ANOVA, factor 1: epoch; factor 2: arm direction, and/or their interaction; $p<0.05$; with Bonferroni corrections for multiple comparisons) revealed that in the large majority of neurons $(85 \%, 127 / 149$, from here on called "task-related cells") the movement execution significantly influenced neural activity in at least one of the three action epochs studied (MOV, HOLD, RET). 
A

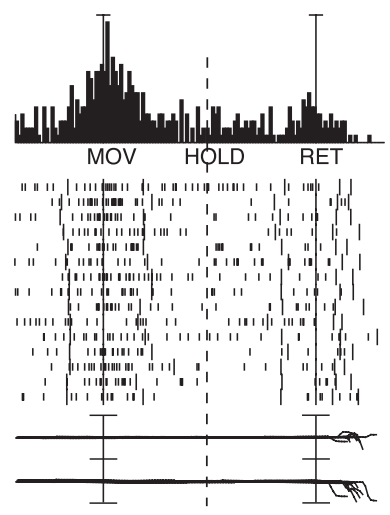

B


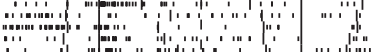

LIGHT


Among task-related cells, significant modulation was observed in 19 cells (15\%) in light conditions, 20 cells (16\%) in dark conditions, and 88 cells (69\%) in both conditions.

The modulating effect of visual conditions on arm-reaching activity and on the spatial tuning of reaching activity will be separately described in the following sections.

\section{Effect of visual conditions on arm-reaching activity}

In $\sim 66 \%$ (85/127) of task-related neurons the presence or absence of vision affected neural activity in epoch MOV (outward reaching movement). Figure 2 shows a cell insensitive to visual conditions (Fig. $2 A$ ) and three examples of visual feedback modulation (Fig. $2 B-D$ ). In all cases, arm movements were directed toward the same spatial position (the central one).

The neuron reported in Figure $2 \mathrm{~A}$ presented a strong modulation of neural activity in epoch MOV, which was not significantly influenced by vision of the moving arm. This behavior indicates that the cell is influenced by proprioceptive input and/or motor corollary discharge, but not visual input at least for the direction of arm movement we tested. In contrast, the neuron illustrated in Figure $2 B$ is activated only during reaching in the light. This neuron displayed a strong discharge for the MOV and the HOLD epochs during reaching in the light, but this discharge disappeared in dark conditions, indicating that reaching-related discharge of this cell is due to visual feedback of the movement. The cell shown in Figure $2 C$ was activated by the arm movement performed in the dark. The neuron's discharge during the reach was stronger in the light, indicating that the effect of visual input summates to the somato-sensory/-motor-related one. Finally, the cell shown in Figure $2 D$ was strongly activated in MOV in the dark, but the modulation completely disappeared in the light. Cells like this are clearly activated by somatosensory and/or movementrelated signals impinging upon the cell during arm movements in dark, but the lack of modulation in light clearly points to an uncommon inhibition of reach-related activity by the visual input.

Vision and absence of vision modulated the activity of taskrelated cells also during the other action epochs of reaching task: $63 \%(80 / 127)$ of cells were affected by visual conditions in epoch HOLD (hand holding), and 56\% (71/127) in epoch RET (in which the arm returned to the home button). Like epoch MOV, also for epochs HOLD and RET the activity could be higher in light or in dark in individual cells.

\section{Effect of visual feedback on spatial tuning of reach-related activity}

A reach-related neuron was defined as "spatially tuned" when it showed a statistically significant difference in mean firing rate in the same action epoch for reaching movement directed toward different spatial locations. Approximately $81 \%$ (103/127) of taskrelated cells were spatially tuned in epoch MOV, 64\% (81/127) in epoch HOLD, and 64\% (81/127) in epoch RET.

The presence or absence of vision affected the spatial tuning of reaching activity differently among cells. Figures 3,4 , and 5 show three examples of this modulation. The activity of unit in Figure 3 was slightly modulated by reaches in the dark, and no signifi-

Figure 2. Effects of vision and absence of vision on reaching activity of four V6A neurons. $A$, Reaching neuron not affected by the availability of visual information. From top to bottom: peri-event time histograms, time epochs, raster displays of impulse activity, recordings of $X$ and $Y$ components of eye position. Long vertical ticks in raster displays are behavioral- or taskrelated markers (see Fig. 1D). Neural activity and eye traces have been aligned twice (on the onset of outward reach movements and on the onset of return movements). Dashed line indicates when trials have been broken (because of different timing across trials) to permit alignment $\leftarrow$

to the two temporal reference points. Scale: Vertical bar on histograms, 60 spikes $/ \mathrm{s}$, eye traces $60^{\circ} \%$ division. $\boldsymbol{B}$, Reaching neuron activated only in the light. Scale: Vertical bar on histograms, 80 spikes/s, eye traces $60 \%$ division. $C$, Reaching neuron more activated in the light than in the dark. Scale: Vertical bar on histograms, 185 spikes $/$, eye traces, $60 \%$ division. $\boldsymbol{D}$, Reaching neuron activated only in the dark. Scale: Vertical bar on histograms, 55 spikes/s, eye traces, $60^{\circ}$ /division. 
A
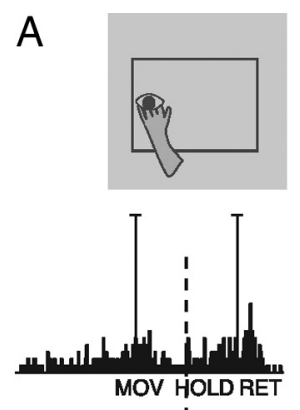

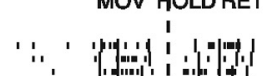

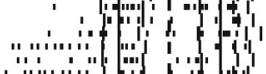



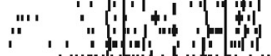

(1.1)
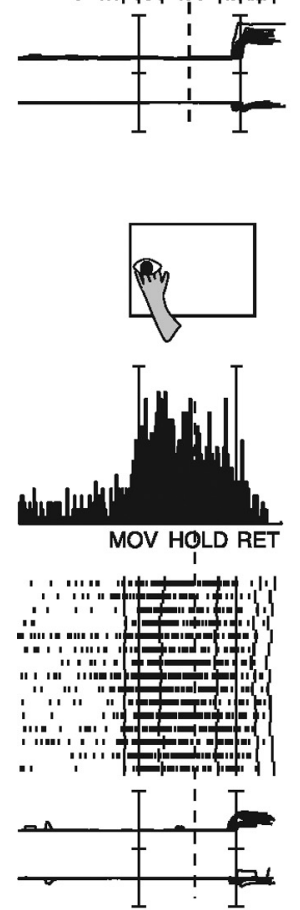

Unit 20,166


Figure 3. Reaching neuron spatially tuned in light. $\boldsymbol{A}$, Schematic illustration of the direction of reaching movement, neural activity and eye traces relative to the corresponding reach directions. Dark is top, light is bottom. All conventions are as in Figure 2. Scale: Vertical bar on histograms, 66 spikes/s: eye traces, $60^{\circ} /$ division. $\boldsymbol{B}$, Plots of the mean activity of the unit shown in $\boldsymbol{A}$ for epochs $\mathrm{MOV}$, HOLD, and RET, respectively, in dark (thick line) and in light (thin line) conditions.

cant differences were found for different target locations (no spatial tuning). However, in the light, the cell strongly discharged for leftward movement, while the activity remained relatively constant for arm movements in other directions. The spike discharge started with the onset of leftward arm movement, continued during hand pressing of the target, and decreased to the resting value when the arm moved back toward the home button. The strong response in light for leftward reaches could be the result of a visual stimulation produced by the arm sweeping across (and remaining within) the visual receptive field of the cell. It is plausible that the receptive field was not stimulated when the arm movement was performed toward the center, or rightward, for the different trajectories followed by the arm in those cases. However, it is interesting to note that in the responsive condition (light, reach to the left target), the cell discharge started before the onset of arm movement, that is when the hand and the arm were entirely outside the visual field of the monkey. Whereas the
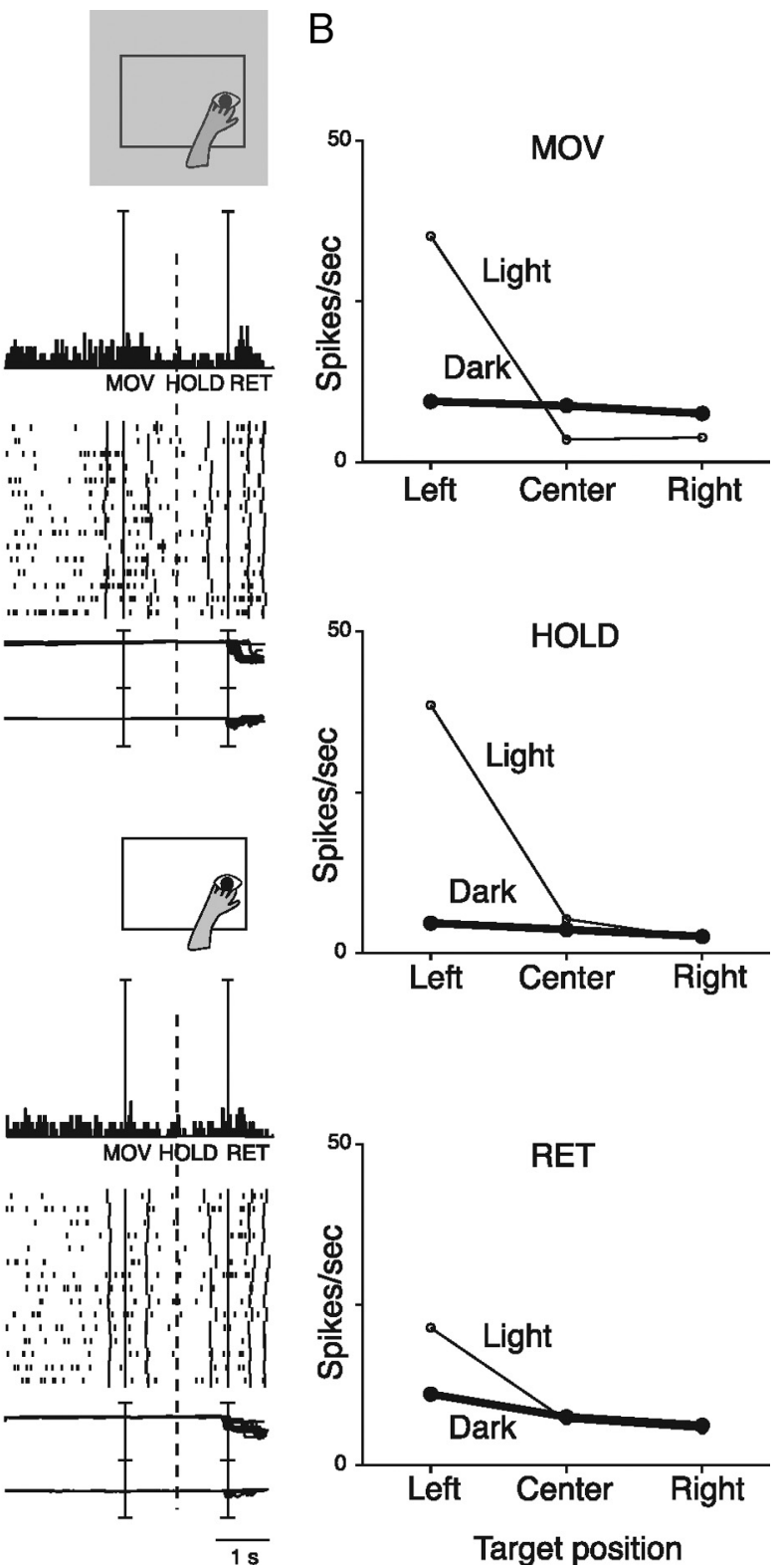

strong neuronal discharge after the onset of the movement may be due to stimulation of visual receptive field by the moving arm, the weak discharge before movement onset may reflect somato-sensory/-motor inputs.

Figure 4 shows a neuron characterized by a strong discharge for leftward and rightward reaching movements in the dark but little or no discharge in the light. As the monkey was executing the same reaching movements in dark and in light, the proprioceptive feedback and/or movement-related corollary discharge would be comparable in the 2 task conditions. Therefore, the only possible interpretation of this bizarre neural behavior is that the presence of a visual feedback cancels out the movement-related discharge.

The unit in Figure 5 presents similar spatial tuning in light and dark conditions: in both cases, rightward arm movements activate the cell more strongly than leftward or central reach movements. In the light, task-related activity was slightly higher than in 
A
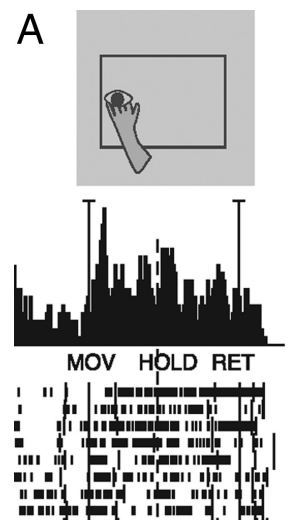

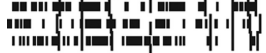
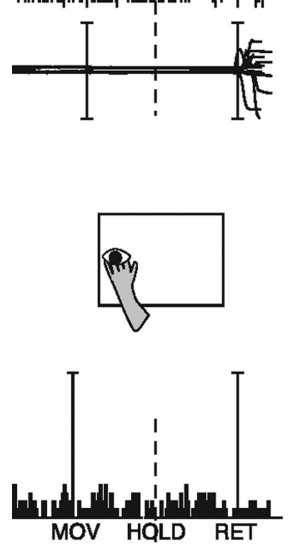

泪明



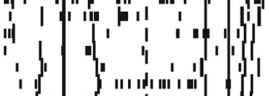

|



Unit 19,112
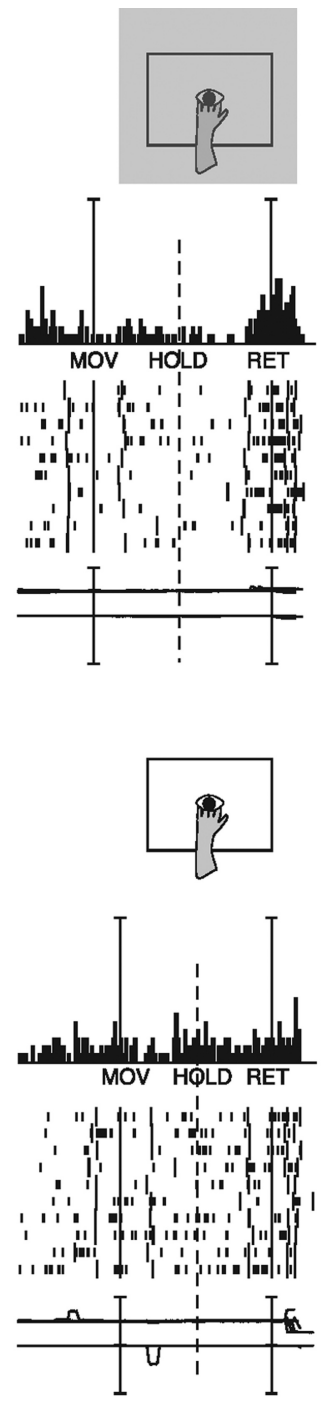
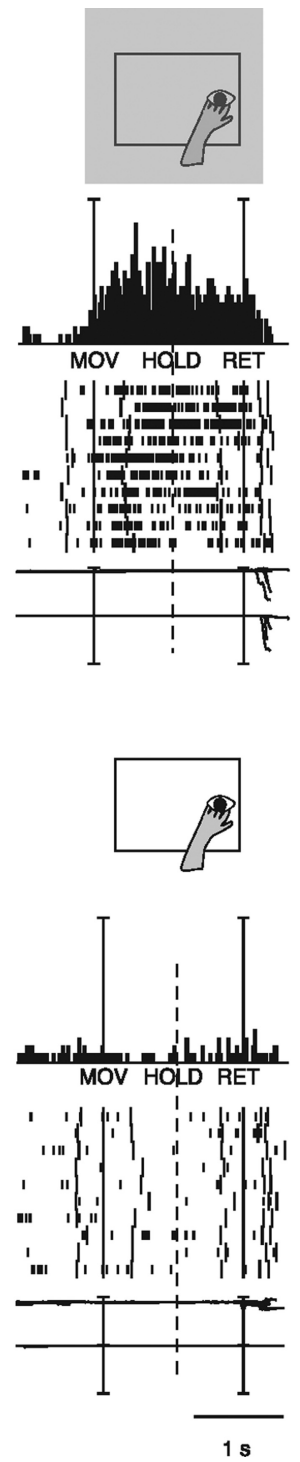

B
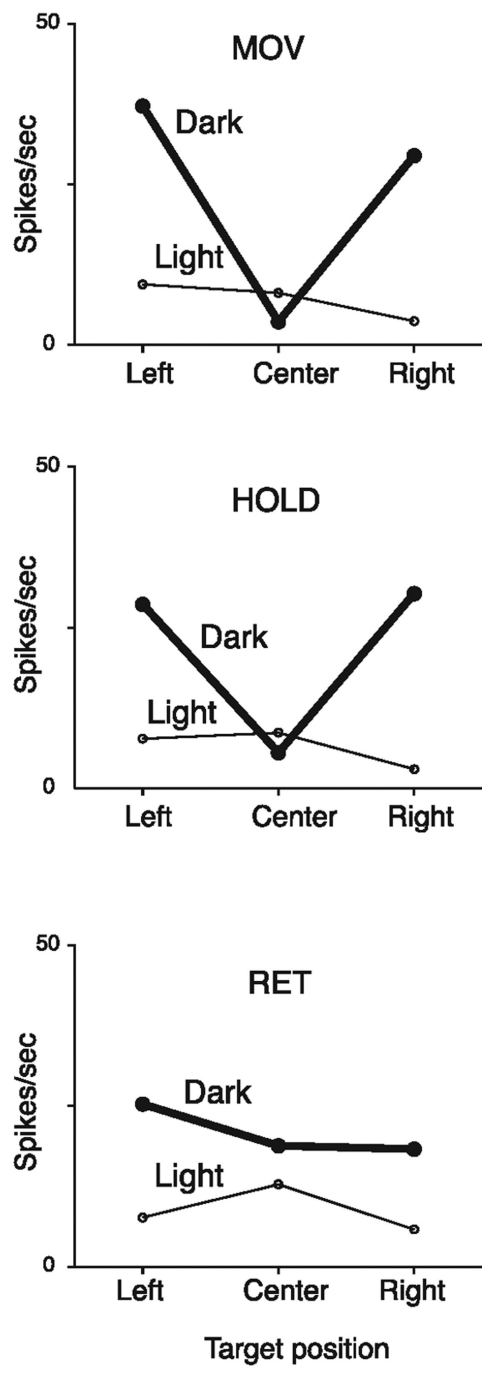

Figure 4. Reaching neuron spatially tuned in dark. A, Neuron spatially tuned in MOV, HOLD and RET, preferring leftward and rightward arm movements in dark (upper part of the figure). All details are as in Figures $3 A$ and 2. Scale: Vertical bar on histograms, 90 spikes/s. $\boldsymbol{B}$, Mean activity, for the same action epochs, of the same unit shown in $\boldsymbol{A}$. Details are as in Figure $3 B$.

dark, particularly for rightward movements. It is likely that this enhancement of cell's response in light is due to excitatory visual inputs converging with extraretinal (somatosensory/efference copy) signals related to the arm movement.

To measure the sensitivity of each neuron to the direction of arm movement in each of the 2 experimental conditions, we calculated a spatial index (SI; see Materials and Methods). The data we obtained are summarized in Figure 6. Each closed circle represents a cell significantly spatially sensitive in one of the two task conditions (bootstrap test, 10.000 iterations, $p<0.05$ ). Symbols located above the diagonal are neurons with spatial sensitivity significantly stronger in light. The reverse is true for symbols located below the diagonal. Cells characterized by a spatial tuning independent of the visual condition (whose bootstrap-estimated confidence interval crosses the diagonal) are represented as crosses.

This analysis shows that the strength of modulation of reaching activity of V6A neurons in light or in dark varies across cells, as also shown in the examples of Figures 3-5. For the epoch MOV, cells with strong spatial tuning in light are more represented than those with strong spatial tuning in dark (36 vs 19).
The opposite was observed for epoch RET (12 vs 27), while in HOLD the incidence was similar (20 vs 16 ).

To investigate whether neurons' light/dark sensitivities change between epochs, we performed the analysis shown in Figure $6 \mathrm{~B}$. Here, in each plot, we show the comparison between pairs of action epochs of the distance from the diagonal for each point in Figure $6 A$. This analysis shows that neurons with a significant spatial index are close to the diagonal in each of the plots, meaning that the spatial index does not change dramatically across epochs.

To quantify how strongly the reaching activity of individual neurons was modulated by the visual input, we used a visual index (VI; see Materials and Methods). The value of VI, which ranges from -1 to 1 , indicates whether the mean reaching activity in light condition is higher (positive value) or lower (negative value) with respect to the dark condition. Figure 7 shows the distribution of VIs of all cells, separately for each action epoch. Neurons more or less excited, or inhibited, by reaching in light assume an approximately Gaussian distribution symmetrical with respect to zero (normal distribution, Cramer-Von Mises, $p<0.05)$. Index values around zero, which are typical of cells not 
A
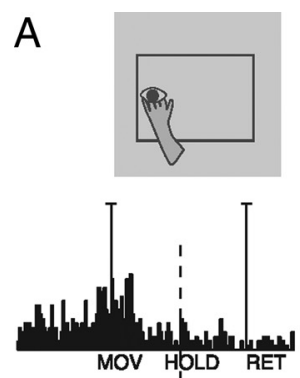


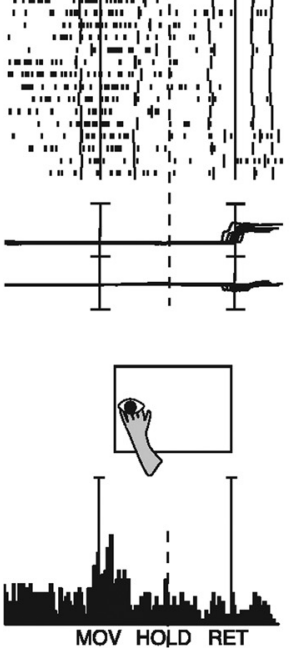

and



Unit 20,131



$1 \mathrm{~s}$

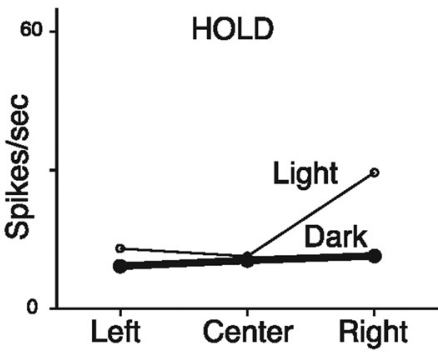

B
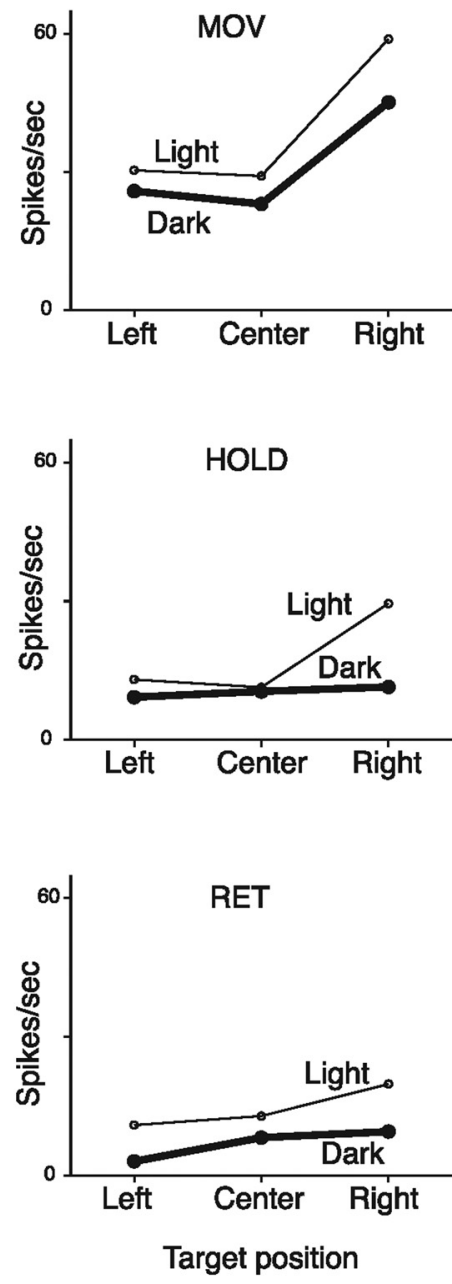

Figure 5. Reaching neuron spatially tuned both in the dark and in the light. $A$, Neuron showing reach-related activity modulated by outward arm movement (MOV) in the light and in the dark. The neuron preferred rightward movements and was weakly modulated, if at all, by task conditions. All details are as in Figures 2 and $3 A$. Scale: Vertical bar on histograms, 110 spikes/s. B, Mean activity, for the same action epochs, of the same unit shown in $\boldsymbol{A}$. All details are as in Figure $3 B$.

affected by visual input, represent the average behavior of cell population. Most of the reaching neurons had VI values significantly different from zero, typical of cells affected by the visual input. Cells excited by the visual input had positive VI values, cells inhibited negative VI values.

The cumulative SDFs of the population of V6A task-related cells shown in Figure 8 show that the activity is on average higher in light than in dark in all action epochs (permutation tests significant for these intervals, $p<0.05$ ), whereas, in the delay period, the activity in the light and in the dark are not significantly different (permutation test, NS).

To check whether visual information could affect the activity during the delay period in reaching neurons modulated in the epoch MOV, we divided them in 2 groups, depending on whether they received inputs of a somatosensory-motor nature (visuomovement- and movement-related neurons) or not (visual neurons).

As Figure 9 shows, in visual cells the activity was higher in the light than in the dark, both during the delay period and the movement period (permutation test for MOV and DELAY, $p<0.05$ ), whereas in the movement-related and visuomovement-related neurons there is no difference in the activity at population level (permutation test, NS for both MOV and DELAY). The behavior of visual cells (Fig. 9A) can be explained by purely "passive" visual inputs impinging on the visual receptive fields well before action execution. Conversely, the data in Figure $9 B$ point to the fact that the visual modulation of visuomovementrelated cells has on average a null effect: the facilitatory and inhibitory effects are quantitatively similar and, at the population level, visuomovement-related cells behave as motor cells. In fact, they discharge similarly in light and dark environment both in the DELAY and in the reaching execution.

A PCA was performed for each action epoch in the two experimental conditions. A normalized representation of the three eigenvectors obtained for each epoch is depicted in Figure 10. Each eigenvector includes three conditions corresponding to the most studied arm directions (ipsilateral, central, and contralateral with respect to the recording side). The relative weights of the eigenvectors, which exemplify their capacity of representing the whole dataset, were obtained normalizing the eigenvalues. In all cases, the sum of the eigenvalues of the first and second principal components is close to or $>95 \%$. This means that the first two ex- 
tracted components account for 95\% of the data variability. In other words, in only $5 \%$ of cases was the response in one of the three positions unpredictable when the activation for the other two positions was known. Thus, two components are almost enough to represent the whole range of the two experimental conditions (light and dark). In a way, this means that the great majority of neurons have a predictable response, most likely monotonic.

To assess whether such figures for the eigenvalues can indicate important trends or whether they are results due to chance from random or nearly random distributions, we generated fictitious activation databases of the same size of the experimental data. In the first simulation, a set of 116 simulated average firing rates have been generated, in which the neural activation in the ipsilateral, central and contralateral positions is fully random. The eigenvalues obtained with a PCA on such data were averaged over 100,000 trials, providing the following figures: $39.0,33.2$, 27.8. It is clear that the second and third eigenvalues have still very high values, supporting the hypothesis that the eigenvalues obtained with our experimental data are very far from being random. In a second simulation, we tried to reduce the randomness of the fictitious activations by giving double probability to the firing rate in either the central or the contralateral positions, and repeated the PCA as above. In both cases, the obtained eigenvalues are 67.0, 18.1, 14.9. Although a clearer prevalence of the first eigenvalue is observed, it is still far from the experimental results, and the third eigenvalue in particular maintains an important effect which was not seen in our data. Moreover, the firing rate distribution of the real population across the three positions (ipsi-centralcontra) is practically homogeneous, so the first simulation is more faithful to the experimental data.

As a second phase of the PCA, we reconstructed the neural activations of each cell using only the first two principal components and computed the difference between the original and the reconstructed activations. Neurons with small differences have most of their variance accounted for by the first two components, and can be considered as "typical," while neurons with high differences are strongly biased by the third component, and we regard them as "outsiders". Studying the characteristics of the two types of neurons, the principal insight that can be drawn is that the typical neurons tend to have a constant activation pattern across conditions. On the contrary, the outsider neurons, that are not easily represented by only two components, are often spatially selective only in the dark or only in the light. This is consistent with the classification of neurons according to their selectivity, shown in the analysis of spatial tuning (see first part of this paragraph).
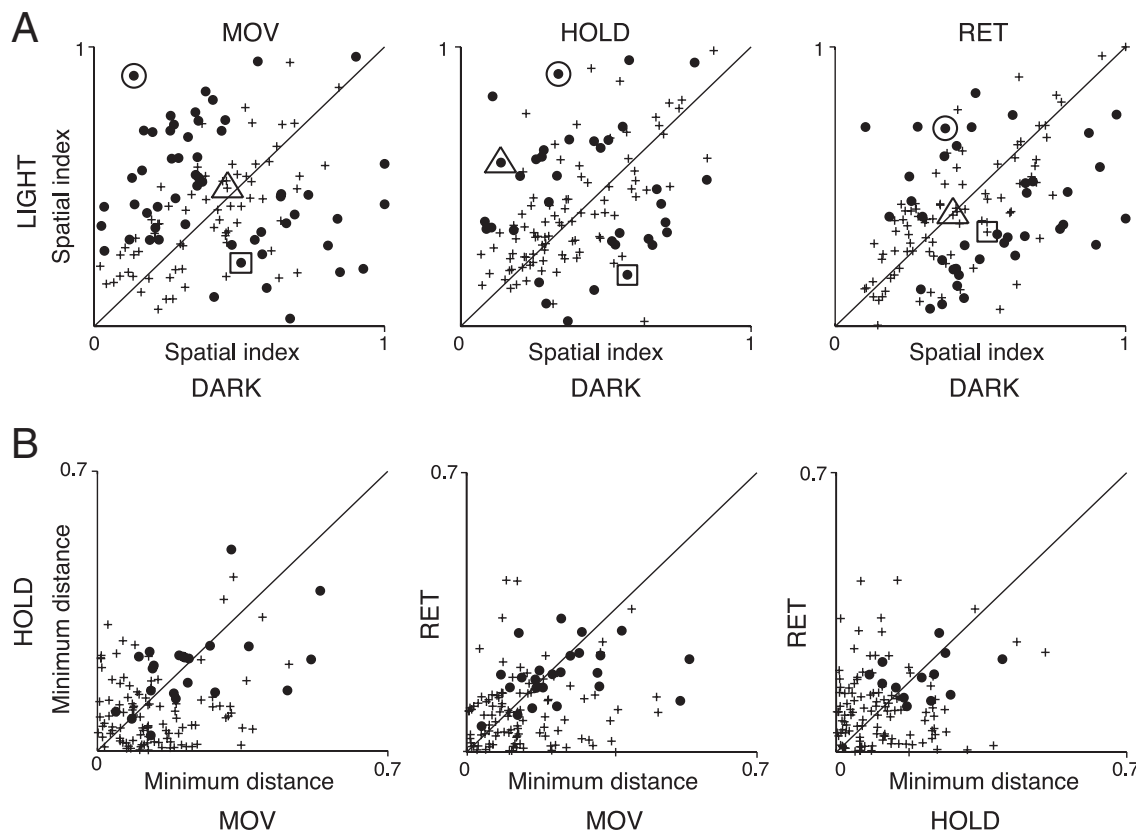

Figure 6. Differences in sharpness of tuning of V6A reaching cells in the two experimental conditions. $\boldsymbol{A}$, Distribution of spatial indices of reaching cells calculated for MOV, HOLD, and RET in dark and light conditions ( $N=127)$. Each point represents one indicate neurons whose bootstrap-estimated confidence intervals cross the diagonal. The big circle, square, and triangle surround points corresponding to the neurons shown in Figures 3, 4, and 5, respectively. $\boldsymbol{B}$, Light/dark sensitivity constancy across action point represents the absolute distance from the diagonal of each neuron shown in $A$. Crosses indicate neuron ppearing as crosses in $\boldsymbol{A}$ in one or both action epochs of the pair; closed circles are neurons significant in both epochs of the pair in
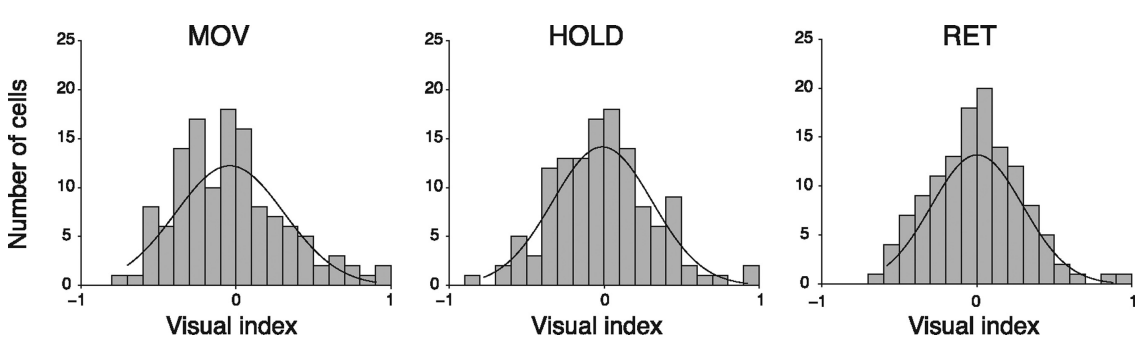

Figure 7. Distribution of visual index in V6A neuronal population. Distributions of visual indices were calculated for each of the action epochs; superimposed on each histogram is shown the best fitting Gaussian curve (Cramer-Von Mises, $p<0.05$ ). Data refer to reach-related activity collected while the animal performed an arm movement directed toward the preferred spatial position in dark. Negative values of the index indicate neurons that present stronger modulation in dark background; positive values neurons that show higher activity in light. Data are plotted for each of the three action epochs considered.

The coefficients that allow to reconstruct neural activations from the eigenvectors were also used to automatically group the neurons in three natural clusters, which numerically confirmed the above observations: the majority of neurons in the first group is spatially selective in one of the two experimental conditions (see examples in Figs. 3, 4). The second group is instead mostly composed by those neurons with spatial selectivity in both light and dark (see example in Fig. 5). Finally, the third group includes those neurons not spatially selective in either condition.

By comparing the PCA in the 2 task conditions, it is evident that the first, dominant component follows approximately the same trend in all epochs. This effect is likely the consequence of a consistent spatial preference in light and dark background. This is confirmed by a direct measure of the phenomenon such as that obtained using the Preferred direction index (PD; see Materials and Methods). Figure $11 \mathrm{~A}$ compares PDs of V6A reach-related cells in dark 




Figure 8. Effect of task conditions (vision and absence of vision) on reaching activity of V6A cells: population behavior. Average SDF of the cells tested in the light (gray line) and in the dark (black line). Preferred directions were taken into account. Each cell was taken into account twice: once when it showed the highest peak activity in light (preferred direction), and another when it showed highest peak activity in dark. The thickness of SDF lines indicates the variability band (SEM). The activity of cells in each plot was aligned twice on the onset of arm movement and on the onset of return movement. Permutation test between light and dark in DELAY, NS; in MOV, HOLD, RET, $p<0.05)$.

\section{VISUAL CELLS MOVEMENT- and VISUOMOVEMENT-}

A

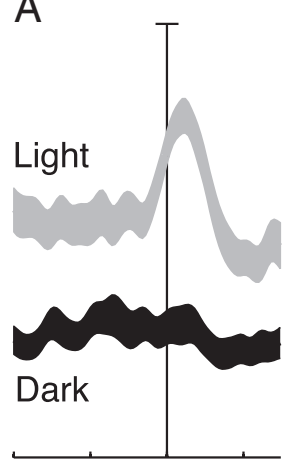

DELAY MOV
$\mathrm{B}$

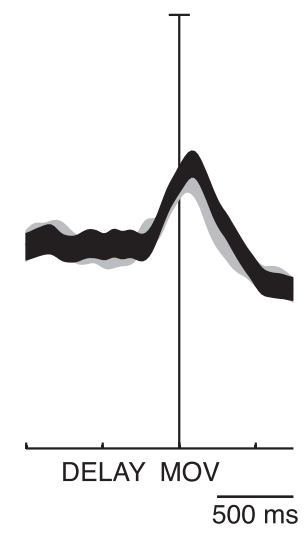

Figure 9. Influence of task conditions between preparation and execution of reaching movements. $\boldsymbol{A}, \boldsymbol{B}$, Visual cells $(\boldsymbol{A})$; visuomovement- and movement-related cells $(\boldsymbol{B})$ : average SDFs of the reaching neurons modulated in light (gray) and in dark (black). Permutation tests: $\boldsymbol{A}$, DELAY, $p<0.05 ;$ MOV, $p<0.05$. B, DELAY, NS; MOV, NS The attribution of the neurons to the two groups has been done based on the visual index (see Fig. 7; visual neurons, $\mathrm{VI} \geq 0.1$; movement-related and visuomovement-related neurons, $\mathrm{Vl}<0.1$ ). All details are as in Figure 8.

and light conditions. The neural activation in all epochs is rather consistent in the two task conditions, and correlation coefficients are $r=0.59$ (MOV), $r=0.54$ (HOLD), and $r=0.67$ (RET). These results suggest that the change in visual conditions weakly influenced the spatial preference of the majority of V6A reaching neurons. There are, however, some exceptions, as the neurons far away from the diagonal in Figure $11 \mathrm{~A}$. In Figure $11 \mathrm{~B}$ there are some examples of reaching cells that change spatial preference between the 2 task conditions.

\section{Functional segregation}

We verified whether cells more sensitive to the arm-reaching movement in a given task condition were segregated within area V6A. Given the possible error in postmortem allocation of the cells which we had recorded from several weeks or months be-
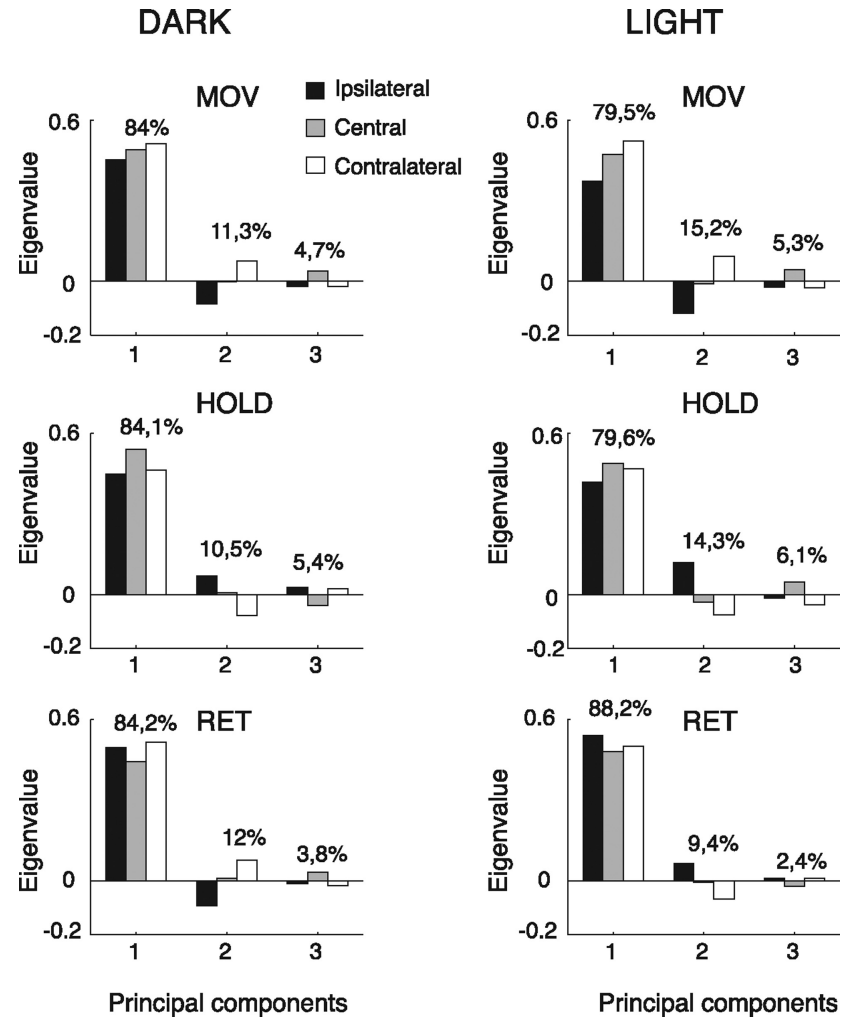

Figure 10. Principal component analysis of reaching movements. Each plot shows the relative weight (in percentage) of the eigenvectors of the three principal components. Each eigenvector includes three conditions corresponding to the ipsilateral (black), central (gray), and contralateral (white) direction of movement with respect to the recording site.

fore, we decided to base our analysis only on the nearby cells recorded from the same microelectrode penetration. Figure 12 shows an example of this type of data. Here, the behavior of three reach-related cells recorded during a single microelectrode penetration in V6A is reported. For each cell, three arm directions were tested for each visual condition. The first neuron discharged more strongly for leftward reaching, and reaching activity was higher in the dark. In the light, the neuron showed weaker activity and was less spatially modulated. Four hundred twenty-four micrometers away we recorded from a second neuron showing opposite spatial preferences with respect to the previous one. It preferred rightward instead of leftward arm movements, and also in this case the reaching activity was higher in the dark. In the light, the spatial tuning of reaching activity was very weak, as for the previous cell. The third neuron, recorded $120 \mu \mathrm{m}$ away from the second one, preferred the light, in contrast with the other two, and showed a different pattern of spatial modulation of reaching activity with respect to the previous two preferring central and leftward arm movements.

In addition to the example of Figure 12, we found 15 penetrations with at least two reaching cells recorded nearby one from another $(<200 \mu \mathrm{m}$ apart $)$, for a total of 32 cells. Among these, the large majority changed the preferred activity in each action epoch according to the visual condition (26/32 in MOV, 28/32 in HOLD and 20/32 in RET), for one or more reach directions. In addition, the majority of these cells changed the preferred arm direction when the task condition was changed. Many cells showed different spatial tuning even when tested with the same task condition (in dark: 24/32 in epoch MOV, 20/32 in epoch HOLD, and 26/32 in epoch RET; in light: 13/32 in MOV, 24/32 in HOLD, and 10/32 
in RET). In conclusion, our data strongly argue against the view that cells with similar spatial tuning of reaching activity and with similar effect of availability or lack of visual information on reaching activity are clustered within area V6A.

\section{Discussion}

V6A is a parietal area receiving visual information (Galletti et al., 1996; 1999; 2001; Gamberini et al., 2009) as well as somatosensory- and movement-related signals relative to reach and grasp actions (Galletti et al., 1997; Fattori et al., 2001; Breveglieri et al., 2002; Fattori et al., 2005; Fattori et al., 2009; Fattori et al., 2010). The present study was designed to determine whether and how the vision of the moving limb influences the activity of V6A neurons during reaching movements.

We studied single cells under two conditions: reaching in the dark and in the light. We assumed that movement-related activity in the light condition may reflect motor efferent copy, as well as proprioceptive and visual afferent feedback. In contrast, activity in the dark condition may reflect only the motor efferent copy and proprioceptive feedback. We found both similarities and differences in neural responses depending on the availability of visual feedback.

The data strongly suggest that vision of the moving arm dramatically changed the reaching activity of many V6A cells (Figs. 3, 4, 6). This was an expected result because the majority of V6A cells are sensitive to visual stimuli and are direction selective (Galletti et al., 1996; 1999), so the moving arm passing across the visual receptive field could strongly modulate the reaching activity. Area V6A has not only central visual field representation, but also a good representation of the periphery. Therefore, it is plausible that greater responsiveness in the light might have been attained with reaches to nonfoveated targets, eliciting the movement of the arm on the visual receptive field.

In a different context, the study of reaches to foveated and nonfoveated targets in the dark has proved that some reaching neurons are organized in retinotopic coordinates, others in spatial coordinates, and still others in a mixed retinotopic/spatial frame of reference. This led us to conclude that V6A is involved in coordinate transformations from a retinotopic to spatial coordinates, a process required for the purposive control of reaching movements (Marzocchi et al., 2008).

In the present experiment we also observed that the preferred direction of movement of the majority of reach-related cells tended to remain constant between dark and light. In other words, the direction of arm movement is encoded in V6A, and this encoding process survives changes in visual information available, as shown by results of PCA and preferred direction (Figs. 10, 11). This aspect can be further investigated in future studies, by allowing the vision of the arm but not of the surroundings, or by occluding the vision of the arm while maintaining the environment illuminated.

As a general insight, the results of our population analysis show that the neural activity during reaching in the light is slightly higher than in the dark (Fig. 8). This is likely the expres- sion of the dominance of visual neurons in V6A ( 60\% of V6A neurons are sensitive to visual stimuli) (Galletti et al., 1996, 1999). Present results are in line with those obtained with 2-deoxyglucose method which demonstrated that in the anterior bank of the parieto-occipital sulcus of the macaque brain, visual-, somesthetic-, and movement-related mechanisms underlie visually guided reaching (Savaki and Dalezios, 1999).

Present results show that visual information influences the preparatory and the execution phases of the reaching in a similar way (Fig. 9A, see activity in DELAY). In fact, population analyses showed that in visual cells the effect of visual information started well before the visual feedback of the moving arm, that is when the arm movement was planned and prepared. We have already reported that V6A shows neural activation in the planning phase of reaching (Fattori et al., 2001) of reach-to-grasp with different wrist orientations (Fattori et al., 2005; 2009; 2010). The neural activity found in the present study during the instructed delay period could be due to generic visual information having nothing to do with visual feedback of the moving limb. Alternatively, it may be related to the anticipation of the consequences of the movement (Kawato, 1999). Further investigation is needed to define the sensorimotor processes and variables encoded by V6A.

\section{Role of V6A in motor control}

We found that the majority of V6A neurons (69\%) showed reach-related activity in both task conditions, while a small number of cells were modulated only in the light (15\%) or only in the dark (16\%). We postulate that some neurons receive only a visual feedback of the arm movement whereas others receive only nonvisual feedback about the arm movement. Unexpectedly, other V6A cells showed a more complex multimodal behavior, such as suppression of nonvisual feedback by light. This poses the question why cells that discharge for reaching in the dark do not discharge for reaching in the light (see examples in Figs. 2D, 4), although motor efferent and somatosensory afferent inputs are the same in both cases. 
A

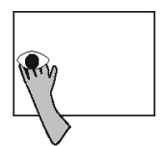

a



b

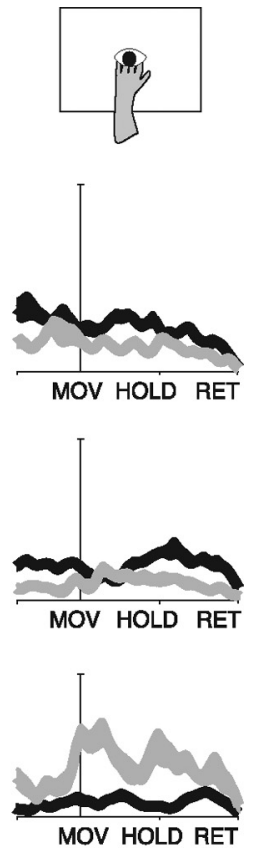



Unit 19.179


Unit 19.181

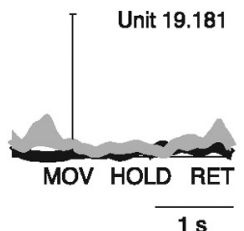

B
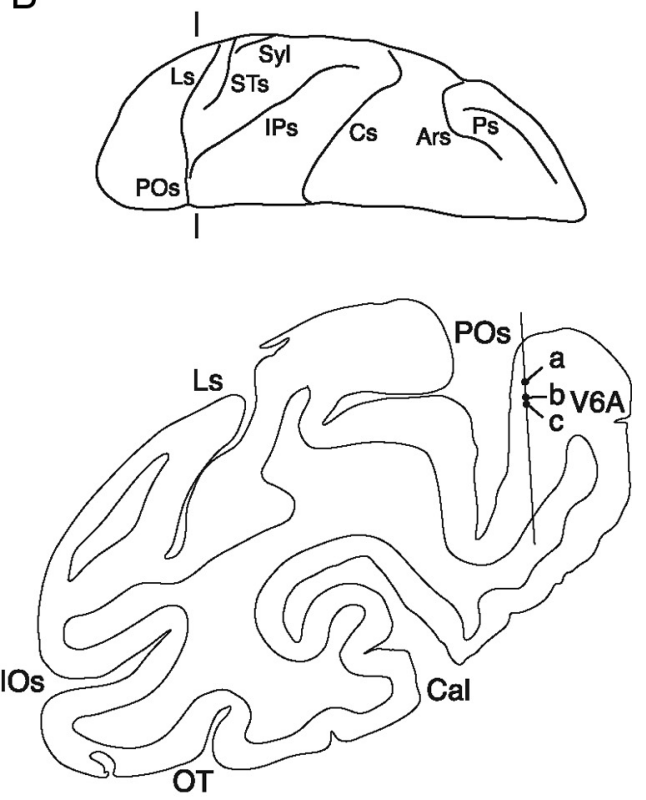

Figure 12. Effect of task conditions on reach-related activity of nearby located V6A cells. $\boldsymbol{A}$, Different effect of arm direction and visual conditions on three nearby reach-related cells $(\boldsymbol{a}-\boldsymbol{c}$ ) recorded along the same microelectrode penetration. On the top, the schematic view of the arm movement direction is reported. Below, the neural activity of the three cells in the dark (black) and in the light (gray) is shown. The neural activity is expressed as SDF with variability band (SEM) and is aligned with the onset of arm movement. $\boldsymbol{B}$, Coronal section taken at the level indicated on the view of the left hemisphere from above. $\boldsymbol{a}-\boldsymbol{c}$, Locations of the three nearby cells recorded along a single microelectrode penetration in V6A cortex. P0s, Pariet0-0ccipital sulcus; Ls, lunate sulcus; IPs, intraparietal sulcus; STs, superior temporal sulcus; Cs, central sulcus; Ars, superior arcuate sulcus; Ps, principal sulcus; Syl, sylvian sulcus.


Movement-related cell

B
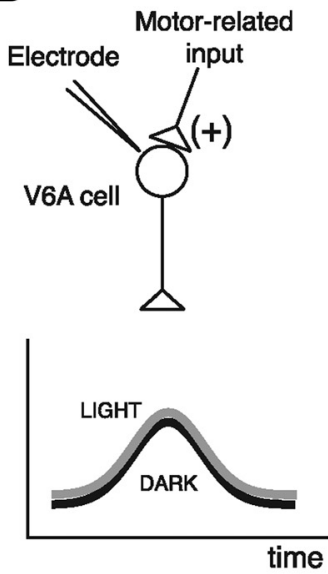

C
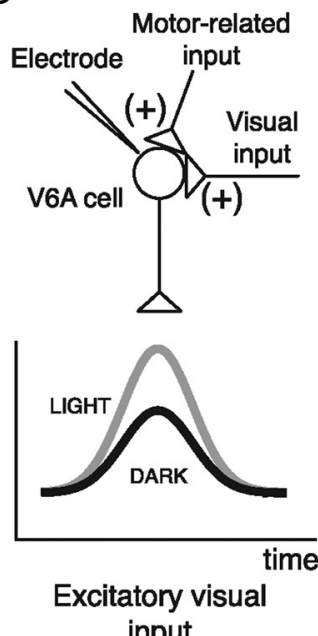

Visuomovement-related cells


Figure 13. Different weight and sign of visual feedback inputs on V6A raching cells. Schematic representation of the inputs impinging upon V6A cells (top) and of their neural activity (bottom).

V6A reaching cells receive two types of inputs, a visual one and a somato-sensory/-motor one related to the arm movement (Galletti et al., 2003). Therefore, three types of cells can be distinguished, a grouping criterion that is also supported by the automatic clustering of the PCA: (1) visual cells (receiving only the visual input) are expected to discharge only in the light (Fig. 13A; see examples in Figs. 2B, 3); (2) movement-related cells (receiving only the afferent somatosensory and/or efferent movement-related information) are expected to show similar intensity of reaching activity in the dark and in the light (see examples in Figs. 2A, 13B); and (3) visuomovement-related cells (receiving both types of inputs) are expected to discharge both in the dark and in the light. If we accept that the visual input could be either excitatory (Fig. 13C) or inhibitory (Fig. 13D), it follows that the visual input could enhance or weaken the reaching activity according to the type of cell taken into account, as in the examples of Figure 2, $C$ and $D$, respectively (see also cells with positive and negative visual index, respectively, in Fig. 7).

The data here reported and the connections of V6A with the frontal cortex (specifically the bidirectional connections with dorsal premotor cortex) (Matelli et al., 1998; Shipp et al., 1998; Marconi et al., 2001; Gamberini et al., 2009) suggest that V6A 
may in part function as "state estimator" in the circuits involved in planning and correctly executing reaching movements (for review, see Grafton, 2010; Shadmehr et al., 2010). V6A could act as comparator between the expected state of the movement, and the visual/somatosensory feedback evoked by the movement. Previous work has shown that V6A reaching activity starts before the earliest electromyographic activity, such that it cannot be entirely explained by afferent somatic signals. It is instead compatible with corollary discharge of motor commands that could reach V6A from the premotor cortex (Fattori et al., 2005). Since the present data demonstrate that visual feedback influences V6A reach-related activity, we suggest that area V6A might compare anticipated and actual sensory feedback evoked by the moving arm. In particular, the visuomovement cells whose activity differs between vision and no-vision conditions could function as an error signal indicating a mismatch between the actual and expected sensory feedback. Although the discovery of such cells, led us to this hypothesis, its confirmation is beyond the scope of this work.

According to this view, V6A and dorsal premotor cortex might form a neural circuit involved in monitoring and correcting the execution of motor actions. The vector from target and hand may be sent by V6A to dorsal premotor cortex, which in turns devises the motor plans required to reach the target. Copy of the resulting motor plans could be sent back, as corollary discharge, to V6A, which might then act as a state estimator, comparing the desired position of the moving limb with the actual one. The former is estimated through forward models of the movement to execute whereas the latter would be monitored through vision and somatosensation (Kawato, 1999; Shadmehr and Krakauer, 2008; Grafton, 2010; Shadmehr et al., 2010). Possible discrepancies may be signaled by the visuomovement cells we report here and be used to adjust the motor plan, so that the ongoing movement keeps in register with the desired one, resulting in an accurate reach.

\section{Comparison with human studies}

Human studies have demonstrated that superior parietal cortex plays a key role in maintaining a continuously updated body representation which codes the current configuration of the body parts, specified in postural coordinates (Head and Holmes, 1911; Critchley, 1953; Pellijeff et al., 2006). The role of SPL in maintaining representations of body state has also been suggested for non-human primates, where it has been reported that the SPL integrates inputs from primary somatosensory cortex to create complex representations of body posture (Sakata et al., 1973) and combines visual and somatosensory signals to monitor limb configuration (Graziano et al., 2000).

A recent study of functional magnetic resonance imaging (fMRI) in humans compared brain effects of visual and nonvisual reaching with saccade (Filimon et al., 2009). Results of this study show that a medial posterior parietal region located at the superior end of parieto-occipital sulcus responds more during visual than non-visual reaches or saccades. Since the activated region is located just anterior to the human area V6 (Pitzalis et al., 2006), and since in the macaque the cortical region anterior to V6 is occupied by area V6A (Galletti et al., 1996; 1999), we suggest that this area could be the human homolog of macaque area V6A (Cavina-Pratesi et al., 2010). Present results, showing that V6A reaching neurons are on average more responsive in the light than in the dark, agree well with this view, suggesting that also human V6A is more responsive to reaching in light.

\section{Conclusions}

Recent findings in macaque have shown an involvement of area V6A in coding the entire act of prehension, from directing the arm toward the object, to adapting wrist orientation to the object orientation, up to the choice of the right grip formation (Fattori et al., 2005; 2009; 2010). It has been proposed that the movementrelated discharges in $\mathrm{V} 6 \mathrm{~A}$ have a role in monitoring the ongoing arm movements during reach-to-grasp actions (Galletti et al., 2003). The present data support the view that V6A cells monitor the ongoing activity taking into account visual information as well as somato-sensory/-motor information. We have shown that the vision of the moving arm modulates reaching activity of V6A neurons in a complex way, increasing or decreasing it according to the type of cell taken into account. We suggest that the contribution of the different cell types found in V6A to the control of reaching movements depends on the availability of sensory information. Some V6A cells may monitor the arm movement on the basis of somato-sensory/-motor inputs, because they do not receive visual input, or their visual inputs are canceled out. Other V6A cells may use the visual information to monitor the ongoing arm movement, as well as hand/object interaction. These data provide empirical support for computational models suggesting task-dependent reweighting of sensory signals dictated by the information content of the visual feedback when the action occurs (Sober and Sabes, 2005; McGuire and Sabes, 2009).

\section{References}

Andersen RA, Snyder LH, Bradley DC, Xing J (1997) Multimodal representation of space in the posterior parietal cortex and its use in planning movements. Annu Rev Neurosci 20:303-330.

Batista AP, Santhanam G, Yu BM, Ryu SI, Afshar A, Shenoy KV (2007) Reference frames for reach planning in macaque dorsal premotor cortex. J Neurophysiol 98:966-983.

Bosco A, Breveglieri R, Chinellato E, Galletti C, Fattori P (2009) Influence of visual feedback on reaching activity in parietal area V6A. Soc Neurosci Abstr 35:355.20.

Breveglieri R, Kutz DF, Fattori P, Gamberini M, Galletti C (2002) Somatosensory cells in the parieto-occipital area V6A of the macaque. Neuroreport 13:2113-2116.

Carlton LG (1981) Visual information: the control of aiming movements. Q J Exp Psychol A 33:87-93.

Cavina-Pratesi C, Monaco S, Fattori P, Galletti C, McAdam TD, Quinlan DJ, Goodale MA, Culham JC (2010) Functional magnetic resonance imaging reveals the neural substrates of arm transport and grip formation in reach-to-grasp actions in humans. J Neurosci 30:10306-10323.

Critchley M (1953) The parietal lobes. New York: Hafner.

Fattori P, Gamberini M, Kutz DF, Galletti C (2001) 'Arm-reaching' neurons in the parietal area V6A of the macaque monkey. Eur J Neurosci 13:2309-2313.

Fattori P, Kutz DF, Breveglieri R, Marzocchi N, Galletti C (2005) Spatial tuning of reaching activity in the medial parieto-occipital cortex (area V6A) of macaque monkey. Eur J Neurosci 22:956-972.

Fattori P, Breveglieri R, Marzocchi N, Filippini D, Bosco A, Galletti C (2009) Hand orientation during reach-to-grasp movements modulates neuronal activity in the medial posterior parietal area V6A. J Neurosci 29:1928-1936.

Fattori P, Raos V, Breveglieri R, Bosco A, Marzocchi N, Galletti C (2010) The dorsomedial pathway is not just for reaching: grasping neurons in the medial parieto-occipital cortex of the macaque monkey. J Neurosci 30:342-349.

Filimon F, Nelson JD, Huang RS, Sereno MI (2009) Multiple parietal reach regions in humans: cortical representations for visual and proprioceptive feedback during on-line reaching. J Neurosci 29:2961-2971.

Galletti C, Battaglini PP, Fattori P (1995) Eye position influence on the parieto-occipital area PO (V6) of the macaque monkey. Eur J Neurosci 7:2486-2501

Galletti C, Fattori P, Battaglini PP, Shipp S, Zeki S (1996) Functional demarcation of a border between areas V6 and V6A in the superior parietal gyrus of the macaque monkey. Eur J Neurosci 8:30-52. 
Galletti C, Fattori P, Kutz DF, Battaglini PP (1997) Arm movement-related neurons in the visual area V6A of the macaque superior parietal lobule. Eur J Neurosci 9:410-413.

Galletti C, Fattori P, Kutz DF, Gamberini M (1999) Brain location and visual topography of cortical area V6A in the macaque monkey. Eur J Neurosci 11:575-582.

Galletti C, Gamberini M, Kutz DF, Fattori P, Luppino G, Matelli M (2001) The cortical connections of area V6: an occipito-parietal network processing visual information. Eur J Neurosci 13:1572-1588.

Galletti C, Kutz DF, Gamberini M, Breveglieri R, Fattori P (2003) Role of the medial parieto-occipital cortex in the control of reaching and grasping movements. Exp Brain Res 153:158-170.

Gamberini M, Passarelli L, Fattori P, Zucchelli M, Bakola S, Luppino G, Galletti C (2009) Cortical connections of the visuomotor parietooccipital area V6Ad of the macaque monkey. J Comp Neurol 513:622-642.

Grafton ST (2010) The cognitive neuroscience of prehension: recent developments. Exp Brain Res 204:475-491.

Graziano MS, Cooke DF, Taylor CS (2000) Coding the location of the arm by sight. Science 290:1782-1786.

Head H, Holmes G (1911) Sensory disturbances from cerebral lesions. Brain 34:102-254.

Hyvärinen J, Poranen A (1974) Function of the parietal associative area 7 as revealed from cellular discharges in alert monkeys. Brain 97:673-692.

Kawato M (1999) Internal models for motor control and trajectory planning. Curr Opin Neurobiol 9:718-727.

Keele SW, Posner MI (1968) Processing of visual feedback in rapid movements. J Exp Psychol 77:155-158.

Kutz DF, Fattori P, Gamberini M, Breveglieri R, Galletti C (2003) Early-and late-responding cells to saccadic eye movements in the cortical area V6A of macaque monkey. Exp Brain Res 149:83-95.

Kutz DF, Marzocchi N, Fattori P, Cavalcanti S, Galletti C (2005) Real-time supervisor system based on trinary logic to control experiments with behaving animals and humans. J Neurophysiol 93:3674-3686. Epub 2005 Feb 3679 .

Luppino G, Hamed SB, Gamberini M, Matelli M, Galletti C (2005) Occipital (V6) and parietal (V6A) areas in the anterior wall of the parieto-occipital sulcus of the macaque: a cytoarchitectonic study. Eur J Neurosci 21: 3056-3076.

Marconi B, Genovesio A, Battaglia-Mayer A, Ferraina S, Squatrito S, Molinari M, Lacquaniti F, Caminiti R (2001) Eye-hand coordination during reaching. I. Anatomical relationships between parietal and frontal cortex. Cereb Cortex 11:513-527.

Marzocchi N, Breveglieri R, Galletti C, Fattori P (2008) Reaching activity in parietal area V6A of macaque: eye influence on arm activity or retinocentric coding of reaching movements? Eur J Neurosci 27:775-789.
Matelli M, Govoni P, Galletti C, Kutz DF, Luppino G (1998) Superior area 6 afferents from the superior parietal lobule in the macaque monkey. J Comp Neurol 402:327-352.

Ma-Wyatt A, McKee SP (2007) Visual information throughout a reach determines endpoint precision. Exp Brain Res 179:55-64.

McGuire LM, Sabes PN (2009) Sensory transformations and the use of multiple reference frames for reach planning. Nat Neurosci 12:1056-1061.

Meyer DE, Abrams RA, Kornblum S, Wright CE, Smith JE (1988) Optimality in human motor performance: ideal control of rapid aimed movements. Psychol Rev 95:340-370.

Mountcastle VB, Lynch JC, Georgopoulos A, Sakata H, Acuna C (1975) Posterior parietal association cortex of the monkey: command function for operations within extrapersonal space. J Neurophysiol 38:871-908.

Pellijeff A, Bonilha L, Morgan PS, McKenzie K, Jackson SR (2006) Parietal updating of limb posture: an event-related fMRI study. Neuropsychologia 44:2685-2690.

Pitzalis S, Galletti C, Huang RS, Patria F, Committeri G, Galati G, Fattori P, Sereno MI (2006) Wide-field retinotopy defines human cortical visual area v6. J Neurosci 26:7962-7973.

Prablanc C, Pélisson D, Goodale MA (1986) Visual control of reaching movements without vision of the limb. I. Role of retinal feedback of target position in guiding the hand. Exp Brain Res 62:293-302.

Sakata H, Takaoka Y, Kawarasaki A, Shibutani H (1973) Somatosensory properties of neurons in superior parietal cortex (area 5) of the rhesus monkey. Brain Res 64:85-102.

Savaki HE, Dalezios Y (1999) ${ }^{14} \mathrm{C}$-deoxyglucose mapping of the monkey brain during reaching to visual targets. Prog Neurobiol 58:473-540.

Shadmehr R, Krakauer JW (2008) A computational neuroanatomy for motor control. Exp Brain Res 185:359-381.

Shadmehr R, Smith MA, Krakauer JW (2010) Error correction, sensory prediction, and adaptation in motor control. Annu Rev Neurosci 33:89-108.

Shipp S, Blanton M, Zeki S (1998) A visuo-somatomotor pathway through superior parietal cortex in the macaque monkey: cortical connections of areas V6 and V6A. Eur J Neurosci 10:3171-3193.

Sober SJ, Sabes PN (2005) Flexible strategies for sensory integration during motor planning. Nat Neurosci 8:490-497.

Vince MA (1948) Corrective movements in a pursuit task. Q J Exp Physiol Cogn Med Sc 1: 85:103.

Wolpert DM, Goodbody SJ, Husain M (1998) Maintaining internal representations: the role of the human superior parietal lobe. Nat Neurosci $1: 529-533$

Woodworth RS (1899) The accuracy of voluntary movement. Psychol Rev Monogr 3:1-106. 